\title{
Introducing Symmetry Conditions in the Integral Equation Technique Applied to Quasi-Waveguide Structures
}

\author{
L. Golestanirad, M. Mattes, J.R. Mosig \\ Laboratory of Electromagnetics and Acoustics \\ Ecole Polytechnique Fédéral de Lausanne \\ Lausanne, Switzerland \\ laleh.golestanirad@epfl.ch
}

\begin{abstract}
Integral Equation (IE) formulation solved by Method of Moments may be used efficiently to address the problem of quasi-waveguide structures, i.e. structures consisting of a main waveguide with a constant cross section that may bear an arbitrary number of printed circuits backed by dielectric substrates. In the case that the structure as well as the excitation has symmetry requirements, simplifications may be applied on the problem leading to a considerable improvement in the usage of computational resources and time. In this paper we have developed and tested the theoretical formulation to apply the symmetry conditions on the IE technique in the context of shielded multilayered structures excited by waveguide ports. The formulation has been successfully applied to waveguide filters which use frequency selective surfaces as coupling elements.
\end{abstract}

Keywords- Integral equation; Method of Moments; multilayerd media; symmetry; waveguide filters;

\section{INTRODUCTION}

Integral equation (IE) technique has been proven to be an efficient tool addressing the problem of printed circuits embedded in shielded multilayered media $[1,2]$. The technique may be efficiently used to model cavity-backed antennas [3] or microwave filters [4] and is also applicable to waveguide structures by applying post processing to retrieve modal scattering parameters.

In the case that the structure as well as the excitation possesses some symmetry properties, simplifications may be applied on the problem to reduce the number of unknowns which leads to a considerable proficiency in the usage of computational resource and time. In this paper we develop a formulation providing the theoretical background for applying symmetry conditions in the context of quasi-waveguide structures, i.e. structures consisting of a main waveguide with a constant cross section that may bear an arbitrary number of printed circuits backed by dielectric substrates. The developed formulation is then applied successfully to waveguide filters based on frequency selective surfaces (FSS).

\section{MATHEMATICAL FORMULATION}

\section{A. Multilayered Media Problem}

The problem of printed circuits embedded in shielded multilayered media has been rigorously examined applying IE technique which is then solved by the Moments Method. The procedure is well described in [3, 5-7] where Green's functions of the media are represented by modal series as:

$$
\overline{\bar{G}}_{P Q}=\sum_{i} P_{i}^{(Q)}\left(z, z^{\prime}\right) \vec{p}_{i}(x, y) \vec{q}_{i}\left(x^{\prime}, y^{\prime}\right)
$$

where $p, q=\{e, h\}$ and $P, Q=\{V, I\}$ which $V$ and $I$ will be obtained using the transmission line model.

Applying symmetry conditions, one comes up with a new boundary value problem which will be solved in the context of waveguide theory and yields another new set of vector modal functions. This set will be then used to represent the Green's functions of the media which is now the truncated geometry.

\section{B. Symmetry}

Generally, an electromagnetic problem may possess three types of planar symmetry [8]: geometric, electric and magnetic. The type of symmetry is defined by the geometric properties of the structure as well as the excitation scheme. If the geometry is symmetric with respect to the symmetry plane, while the sources are arbitrarily located the current/field distribution will not generally possess any symmetric properties and the unknown coefficients on the whole mesh must be solved and therefore no reduction in memory usage will be obtained. In the case of electric symmetry, not only geometric symmetry holds but also additional requirement are met by the sources in the way that tangential component of electric field and normal component of magnetic field disappear at the symmetry plane, allowing replacing it by a PEC wall. In a similar way, in the case of magnetic symmetry the source distribution guarantees that the tangential component of magnetic field and the normal

This work has been partially supported by Thales Group. 
component of electric field vanish on the symmetry plane and so allow replacing it with a PMC wall.

In the framework of boxed multilayered media applying symmetry simplifications will result in modifications of the boundary conditions of the longitudinal problem. To illustrate the problem let's consider the general case of a rectangular waveguide problem as depicted if Fig. 1. As we know [9] $T M_{z}$ fields can be expressed as:

$$
\begin{gathered}
E_{x}=\frac{1}{j \omega \varepsilon} \frac{\partial^{2} \psi}{\partial x \partial z} E_{y}=\frac{1}{j \omega \varepsilon} \frac{\partial^{2} \psi}{\partial y \partial z} E_{z}=\frac{1}{j \omega \varepsilon}\left(\frac{\partial^{2} \psi}{\partial z^{2}}+k^{2}\right) \psi \\
H_{x}=\frac{\partial \psi}{\partial y} H_{y}=-\frac{\partial \psi}{\partial x} \quad H_{z}=0
\end{gathered}
$$

And for the $T E_{z}$ fields we have:

$$
\begin{gathered}
H_{x}=\frac{1}{j \omega \mu} \frac{\partial^{2} \psi}{\partial x \partial z} H_{y}=\frac{1}{j \omega \mu} \frac{\partial^{2} \psi}{\partial y \partial z} H_{z}=\frac{1}{j \omega \mu}\left(\frac{\partial^{2} \psi}{\partial z^{2}}+k^{2}\right) \psi \\
E_{x}=-\frac{\partial \psi}{\partial y} \quad E_{y}=\frac{\partial \psi}{\partial x} \quad E_{z}=0
\end{gathered}
$$

in which $\psi$ is the potential function satisfying Helmholtz's equation subject to the appropriate boundary conditions which in the case of perfect electric walls lead to the conventional $T E_{z}$ and $T M_{z}$ rectangular waveguide modes. Now consider that the structure has a symmetric geometry with respect to $\mathrm{x}$ and $\mathrm{y}$ axes and also the incident wave distribution allows assuming the symmetric planes along both axes in such a way that the structure can be split by introducing two symmetric planes. For example, if the waveguide is excited by its fundamental mode we may introduce a horizontal PEC and a vertical PMC plane as depicted in Fig.1a. The original problem is then converted to a new waveguide problem subject to the new boundary conditions such as the tangential component of electric field and magnetic field vanishes on PEC and PMC walls, respectively. Applying these criteria to equations (2) yields the boundary conditions that must be satisfied by the potential function $\psi$ for $T E_{z}$ and $T M_{z}$ waves, as illustrated in Fig. $1 \mathrm{~b}$ and Fig. 1c.

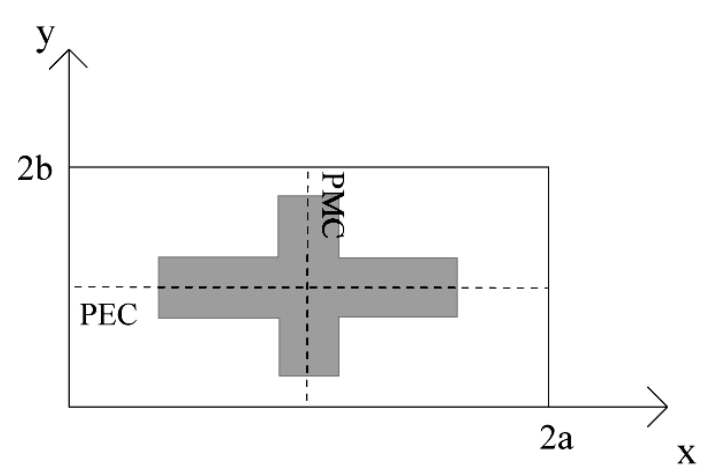

Figure 1. a: The general geometry of a symmetric waveguide structure

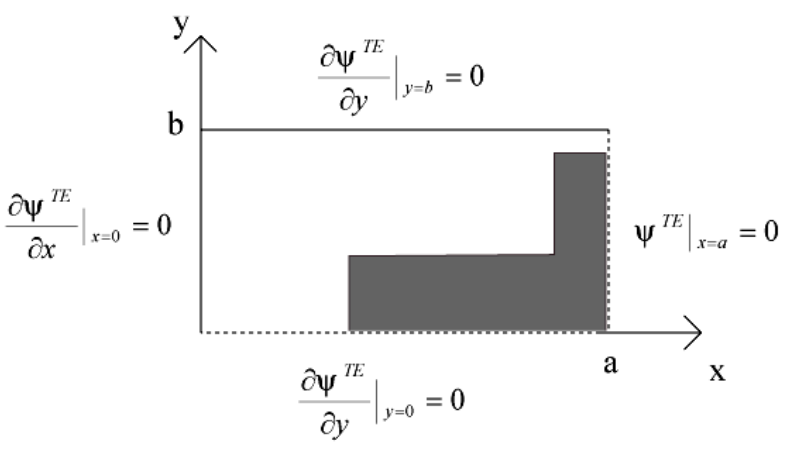

Figure 1. b: the solution of Helmholtz's equation for TE modes subject to the symmetry boundary conditions

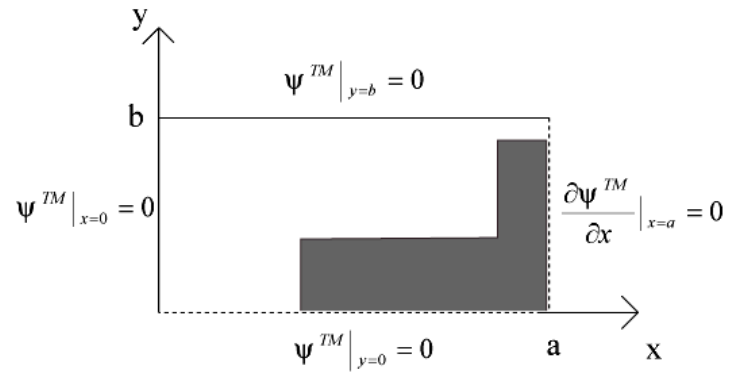

Figure 1. c: the solution of Helmholtz's equation for TM modes subject to the symmetry boundary conditions

Solving this boundary value problem in the Cartesian coordinate, one can obtain the potential functions for $T E_{z}$ and $T M_{z}$ waves as:

$$
\begin{aligned}
& \psi_{m n}^{T M}=A_{m n} \sin \left(\frac{2 m-1}{2 a} \pi x\right) \sin \left(\frac{n}{b} \pi y\right) \\
& \psi_{m n}^{T E}=A_{m n} \cos \left(\frac{2 m-1}{2 a} \pi x\right) \cos \left(\frac{n}{b} \pi y\right)
\end{aligned}
$$

These scalar potentials will be used to obtain the vector modal functions of the waveguide structure which represent the Green's functions of layered media:

$$
\vec{e}_{m n}^{T E}=\hat{z} \times \nabla_{t} \psi_{m n}^{T E} / k_{c m n} \quad \vec{e}_{m n}^{T M}=-\nabla_{t} \psi_{m n}^{T M} / k_{c m n}
$$

and

$$
\vec{h}_{m n}=\hat{z} \times \vec{e}_{m n}
$$

\section{NumericAl RESUlts}

To verify the results of the abovementioned formulation we have used a waveguide filter made up by three frequency selective surfaces (FSS) whose full geometry is depicted in Fig. 2. Each FFS is a dual-behavior resonator by the combination of the aperture-type and the patch-type FSSs as presented in [10] whose geometry is depicted in Fig. 3. The geometry details of each resonator are given in Fig. 2b. All the design parameters have been chosen as proposed in [10]. The structure has been 
modeled by a quarter of it using two symmetry planes. Note that when modeling the quarter of the structure, we have to define half-rooftops or half-RWGs on the cutting edges (Fig. 4) in order to model the flux of current which physically is not zero at these artificially introduced ending edges. The same strategy should be adopted modeling the wave ports when magnetic currents are introduced on them.

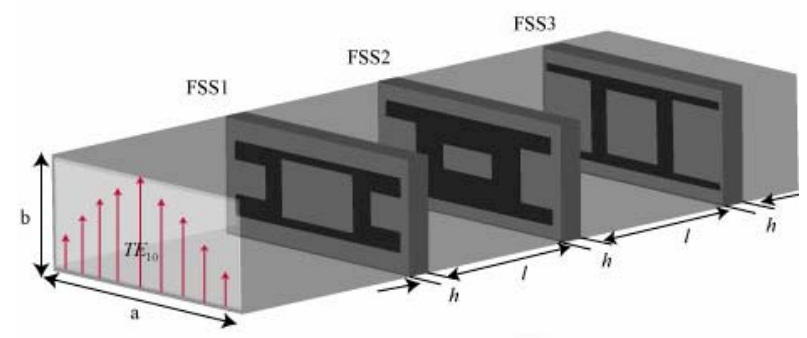

Figure 2. Waveguide filter with frequency selective surfaces (a) FSS1 $\left(x_{1}=\right.$ $\left.0.34, \mathrm{x}_{2}=3.99, \mathrm{y}_{1}=2.77, \mathrm{y}_{2}=1.57,1_{1}=8.8,1_{2}=4.26\right)$, (b) FSS2 $\left(\mathrm{x}_{1}=0.3, \mathrm{x}_{2}=\right.$ $\left.3.36, \mathrm{y}_{1}=0.81, \mathrm{y}_{2}=1.41, \mathrm{1}_{1}=8.8, \mathrm{l}_{2}=2.52\right)$, (c) FSS3 ( $\mathrm{x}_{1}=0.56, \mathrm{x}_{2}=5.4, \mathrm{y}_{1}=$ $0.77, \mathrm{y}_{2}=0.46,1_{1}=5.67,1_{2}=5.63$ )

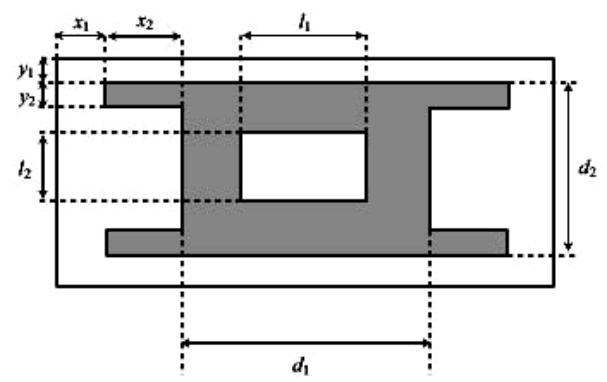

Figure 3. The element shaspe

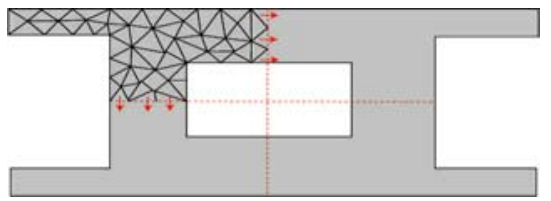

Figure 4. Defining basis functions to allow for the flux of current through the cutting edges

The scattering parameters are presented in Fig. 5 which shows a fair agreement with HFSS results. The spectrum is slightly shifted toward lower frequencies which is mostly due to the triangular mesh which forces the currents flow along longer paths than the actual one. As it is observed, this effect is more pronounceable in higher frequencies.

\section{CONCLUSION}

The symmetry boundary condition has been successfully developed and applied in the analysis of quasi-waveguide structures using IE technique solved by Method of Moments in the context of laterally shielded multilayered media. This provides a considerable gain in the terms of computational resources and time.

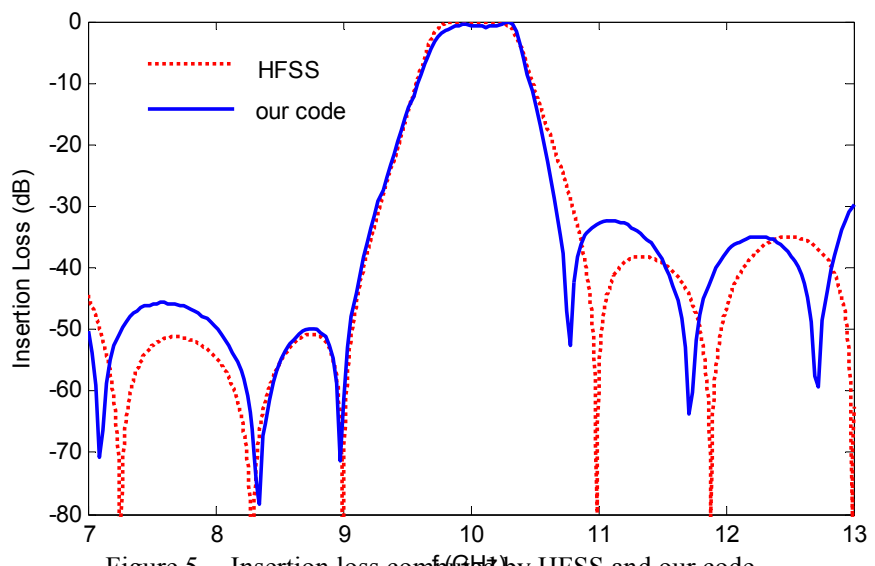

Figure 5. Insertion loss comfl(4t) by HFSS and our code

\section{REFERENCE}

J. R. Mosig, "Arbitrarily shaped microstrip structures and their analysis with a mixed potential integral equation," Microwave Theory and Techniques, IEEE Transactions on, vol. 36, pp. 314323, 1988.

J. R. Mosig, "Integral-equation technique," in Numerical Techniques for Microwave and Millimeter-Wave Passive Structures, T. Itoh, Ed. New York: Wiley, 1989, pp. 133-213.

T. Grzegorczyk, "Integrated 3D Antennas for Millimeter-wave applications: theoretical study and Technological Realization," in Laboratory of Electromagnetics and Acoustic. vol. PhD Lausanne: Ecole Polytechnique Fédéral de Lausanne, 2000.

P. Crespo-Valero, "Electromagnetic modeling of planar circuits in bounded layered medida," in Laboratory of Electromagnetics and Acoustic vol. PhD Lausanne: Ecole Polytechnique Fédéral de Lausanne, 2007.

M. M. Pedro Crespo-Valero, Ivica Stevanovic, "A numerically stable transmission line model for multilayered Green's functions," in Antennas and Propagation Society International Symposium, 2005, pp. 200-203.

[6] M. M. Pedro Crespo-Valero, Ivica Stevanovic, Juan Mosig, "Analysis of Multilayer Boxed Printed Circuits," in IEEE Mediterranean Electro technical Conference, 2006, pp. 206-209.

A. Alvarez Melcon, "Application of the Integral Equation Technique to the Analysis and Synthesis of Multilayered Printed Shielded Microwave Circuits and Cavity Backed Antennas." vol. PhD: Ecole Polytechnique Fédéral de Lausanne, 1998.

[8] "Symmetry options in FEKO, "http://www.feko.info/feko-productinfo/technical/special-module-and-feature-articles/symmetry,

[10] M. Ohira, H. Deguchi, M. Tsuji, and H. Shigesawa, "Novel waveguide filters with multiple attenuation poles using dualbehavior resonance of frequency-selective surfaces," Microwave Theory and Techniques, IEEE Transactions on, vol. 53, pp. 33203326,2005 . 